\title{
Inaugural Young Investigator Issue for Cells Tissues Organs
}

\author{
Ryan J. Gilbert ${ }^{a}$ Guojun Sheng $^{b}$ Christoph Viebahn $^{c}$ Stefan Liebau $^{d}$ \\ Kacey G. Marra ${ }^{\mathrm{e}}$ Loredana De Bartolo $^{f}$ \\ ${ }^{a}$ Center for Biotechnology and Interdisciplinary Studies, Department of Biomedical Engineering, Rensselaer \\ Polytechnic Institute, Troy, NY, USA; 'b International Research Center for Medical Sciences, Kumamoto University, \\ Kumamoto, Japan; Institute of Anatomy and Embryology, University Medical Center Gottingen, Gottingen, \\ Germany; Institute of Neuroanatomy and Developmental Biology, University of Tübingen, Tübingen, Germany; \\ eDepartments of Plastic Surgery and Bioengineering, University of Pittsburgh, Pittsburgh, PA, USA; Institute of \\ Membrane Technology, National Research Council of Italy, Rome, Italy
}

It is with great enthusiasm we present the inaugural Young Investigator issue for the journal Cells Tissues Organs (CTO). The genesis of the Young Investigator issue initiative began with our desire to introduce CTO to emerging, independent investigators in the journal's emphasis areas of developmental biology, stem cell biology, and tissue engineering and regenerative medicine. We also sought to select investigators pursuing research in new areas of investigation that would resonate well with the journal's readership. During our review of submitted applications, we found it challenging to select the best applicants, as we were very impressed with the applicant quality and interesting topics they proposed. In the end, the awardees selected highlight promising new investigators with tremendous potential in fascinating areas of investigation. We congratulate all of the awardees and look forward to their upcoming research endeavors.

The awardees submitted either an original research manuscript or a review manuscript in their areas of investigation for the Young Investigator issue. The following summarizes the Young Investigator awardee's contributions.
From Dr. Mohit Jolly's laboratory at the Centre for BioSystems Science and Engineering at the Indian Institute of Science in Bangalore, India, a manuscript titled "A Computational Systems Biology Approach Identifies SLUG as a Mediator of Partial Epithelial-Mesenchymal Transition (EMT)" presents the capability of computational approaches to identify regulators of epithelial-mesenchymal plasticity [Subbalakshmi et al., 2021]. Using these approaches, Dr. Jolly and colleagues found that SNAIL is a stronger inducer of complete EMT than SLUG. However, SLUG (a transcriptional repressor of E-cadherin) can alter the mean residence time of cells in a hybrid E/M phenotype and high SLUG expression correlates with poor cancer prognosis. Such improved understanding of the role of SLUG may lead to a better understanding of drug resistance and immune evasion across different cancer types.

From Dr. Natasha Chang's laboratory at the Department of Biochemistry at McGill University in Montreal, Canada, a manuscript titled "Empowering Muscle Stem Cells for the Treatment of Duchenne Muscular Dystrophy" presents a review discussing the potential of restoring endogenous muscle stem cell function as a regenera- karger@karger.com www.karger.com/cto
(C) 2021 S. Karger AG, Base
Correspondence to:

Ryan J. Gilbert, gilber2@ rpi.edu 
tive medicine strategy to mitigate Duchenne Muscular Dystrophy (DMD) [Filippelli and Chang, 2021]. In the review, Dr. Chang and Ms. Filippelli propose that satellite stem cell restoration therapy should be considered in combination with current approaches that are used to restore dystrophin protein levels for individuals with DMD. They suggest that a more holistic, combinatorial approach is required to produce better patient outcomes.

From Dr. Peter Walentek at the Center of Integrative Biological Signaling Studies at the University of Freiburg, Germany, a manuscript titled "Signaling Control of $\mathrm{Mu}$ cociliary Epithelia: Stem Cells, Cell Fates, and the Plasticity of Cell Identity in Development and Disease" presents a review summarizing the major findings and provides future perspectives on mucociliary research with regards to the treatment of chronic airway diseases [Walentek, 2021]. In this review, Dr. Walentek suggests that there are many links between Wnt, Notch, BMP, and other signaling pathways related to airway epithelia and that further investigation of these links may lead to new treatments for airway infections and chronic lung diseases.

From Dr. Ryan Stowers at the Department of Mechanical Engineering at the University of California, Santa Barbara, USA, a manuscript titled "Advances in Extracellular Matrix-Mimetic Hydrogels to Guide Stem Cell Fate” presents a review summarizing recent advances in materials to guide stem cell fate with a focus on innovative methods to capture extracellular matrix functionalities [Stowers, 2021]. Such advances in material design may lead to the production of distinct materials capable of better understanding stem cell biology or leading to material/cellular systems capable of providing therapy following injury or treatment for disease.

From Dr. Courtney Dumont's laboratory at the Department of Biomedical Engineering at the University of Miami, FL, USA, a manuscript titled "Biomaterial Strategies to Bolster Neural Stem Cell-Mediated Repair of the Central Nervous System" presents a review on the importance of developing materials capable of protecting stem cells implanted into injured central nervous system [Tedada et al., 2021]. Dr. Dumont and colleagues state that these materials should be designed to also control the cytotoxic environment at the injury site to promote implanted stem cell longevity.

From Dr. Liliana Moreira Teixeira's laboratory at the Department of Developmental Bioengineering at the Technical Medical Centre, University of Twente, the Netherlands, a manuscript titled "New Endeavors of (Micro)Tissue Engineering: Cells Tissues Organs on-Chip and Communication Thereof" presents a review of the promise of organs-on-chips (OoC) technology [Ahmed and Moreira Teixeira, 2021]. Specifically, Dr. Moreira Teixeira and colleagues describe key examples of multiorgan/tissue-on-chip approaches or linked organs/tissues-on-chip and describe the challenges and potential applications of advanced in vitro model systems.

From Dr. Riccardo Gottardi's laboratory at the Department of Pediatrics at the University of Pennsylvania and Children's Hospital of Philadelphia, USA, an original research manuscript titled "A High-Throughput Mechanical Activator for Cartilage Engineering Enables Rapid Screening of in vitro Response of Tissue Models to Physiological and Supra-physiological Loads" presents development of a device that enables high-throughput compressive loading of tissue constructs [Capuana et al., 2021]. The HighThroughput Mechanical Activator for Cartilage Engineering (HiT-MACE) device was used to study cyclic loading in physiological and supra-physiological situations using an in vitro model of cartilage osteoarthritis.

In closing, we again congratulate the inaugural Young Investigator issue awardees. We and Karger Publishers remain committed to the Young Investigator award initiative. We look forward to next year's Young Investigator competition where we will again seek to highlight outstanding young investigator accomplishments and present new research in the areas of developmental biology, stem cell biology, and tissue engineering and regenerative medicine.

\section{Conflict of Interest Statement}

The authors have no conflicts of interest to declare.

\section{Funding Sources}

None.

\section{Author Contributions}

Ryan J. Gilbert drafted the original text. Guojun Sheng, Christoph Viebahn, Stefan Liebau, Kacey G. Marra, and Loredana De Bartolo provided comments to the original draft and supported the Young Investigator award initiative. 


\section{References}

Ahmed HMMAM, Moreira Teixeira LS. New endeavors of (micro)tissue engineering: cells tissues organs on-chip and communication thereof. Cells Tissues Organs. 2021. doi: $10.1159 / 000516356$.

Capuana E, Marino D, Di Gesù R, La Carrubba V, Brucato V, Tuan RS, et al. A high-throughput mechanical activator for cartilage engineering enables rapid screening of in vitro response of tissue models to physiological and supraphysiological loads. Cells Tissues Organs. 2021. doi: 10.1159/000514985.
Filippelli RL, Chang NC. Empowering muscle stem cells for the treatment of Duchenne Muscular Dystrophy. Cells Tissues Organs. 2021. doi: 10.1159/000514305.

Stowers RS. Advances in extracellular matrix-mimetic hydrogels to guide stem cell fate. Cells Tissues Organs. 2021.doi: 10.1159/000514851.

Subbalakshmi AR, Sahoo S, Biswas K, Jolly MK. A computational systems biology approach identifies SLUG as a mediator of partial epithelial-mesenchymal transition (EMT). Cells Tissues Organs. 2021.doi:10.1159/000512520.
Tedada G, Ciciriello AJ, Dumont CM. Biomaterial strategies to bolster neural stem cell-mediated repair of the central nervous system. Cells Tissues Organs. 2021. doi: 10.1159/000515351.

Walentek P. Signaling control of mucociliary epithelia: stem cells, cell fates, and the plasticity of cell identity in development and disease. Cells Tissues Organs. 2021. doi: $10.1159 / 000514579$. 